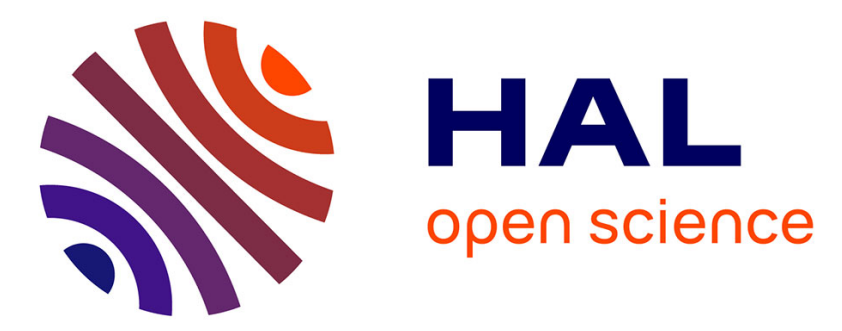

\title{
Explanation of the anomalous enhancement effect observed in single crystal neutron diffraction of $\alpha$-LiIO3 : \\ a new method to determine the activation energy of $\alpha$-LiIO3 by this effect \\ Hua-Chen $\mathrm{Hu}$
}

\section{To cite this version:}

Hua-Chen Hu. Explanation of the anomalous enhancement effect observed in single crystal neutron diffraction of $\alpha$-LiIO3 : a new method to determine the activation energy of $\alpha$-LiIO3 by this effect. Journal de Physique Lettres, 1981, 42 (9), pp.189-192. 10.1051/jphyslet:01981004209018900 . jpa00231906

\section{HAL Id: jpa-00231906 https://hal.science/jpa-00231906}

Submitted on 1 Jan 1981

HAL is a multi-disciplinary open access archive for the deposit and dissemination of scientific research documents, whether they are published or not. The documents may come from teaching and research institutions in France or abroad, or from public or private research centers.
L'archive ouverte pluridisciplinaire HAL, est destinée au dépôt et à la diffusion de documents scientifiques de niveau recherche, publiés ou non, émanant des établissements d'enseignement et de recherche français ou étrangers, des laboratoires publics ou privés. 


\title{
Explanation of the anomalous enhancement effect observed in single crystal neutron diffraction of $\alpha-\mathrm{LiIO}_{3}$ : a new method to determine the activation energy of $\alpha$-LiIO $\mathrm{O}_{3}$ by this effect
}

\author{
$\mathrm{Hu}$ Hua-Chen \\ Institute of Atomic Energy, Academia Sinica, Beijing, China
}

(Reçu le 4 décembre 1980, accepté le 23 mars 1981)

\begin{abstract}
Résumé. - Ce travail donne une interprétation de la diffraction par $\alpha-\mathrm{LiIO}_{3}$ basée sur la théorie des dipôles élastiques. L'analyse du processus dynamique montre de façon originale que l'on peut obtenir deux énergies d'activation de diffusion de défauts chargés dans le cristal à partir de la courbe de relaxation de l'intensité.

Abstract. - This work gives an explanation of the anomalous neutron diffraction effect on $\alpha-\mathrm{LiIO}_{3}$ based on elastic dipole theory and shows that by analysing the dynamical processes one can obtain two diffusion activation energies of charged defects within the crystal from the intensity relaxation curve, a new method which has not been used before.
\end{abstract}

Yang Zhen et al. [1-3] reported an anomalous new effect observed in the single crystal neutron diffraction of $\alpha-\mathrm{LiIO}_{3}$ : the peak intensities in the $(00 l)$ plane can be enhanced to several times their normal value with a moderate voltage applied along the crystallographic $c$-axis. The main features of this effect include the following. (1) It is very sensitive, e.g., in some cases a mere $4 \mathrm{~V} / \mathrm{cm}$ field strength can induce a $13 \%$ increase in intensity, and a 14 fold increase in intensity can be produced with a $160 \mathrm{~V} / \mathrm{cm}$ field strength. (2) It is highly anisotropic : while all the $(00 l)$ group planes show a very distinct enhancement with the field along the $c$-axis, the (010) and (100) planes show no change within the statistical fluctuations $(\sim 1 \%)$ with $E_{\mathrm{c}}=6000 \mathrm{~V} / \mathrm{cm}$. (3) The rocking curve of the enhanced $(00 l)$ peak is slightly broadened when the applied voltage is high $\left(E_{\mathrm{c}}>120 \mathrm{~V} / \mathrm{cm}\right)$. (4) The enhancement effect is accompanied by a complex behaviour of the relaxation process; the relaxation time varies inversely with sample temperature and approaches infinity when the sample temperature drops to $\sim 170 \mathrm{~K}$ (the so-called "freezing effect »). (5) A low frequency AC field also induces diffraction intensity enhancement ; the magnitude of the enhancement varies inversely with frequency and disappears at about $1 \mathrm{kC} / \mathrm{s}$. All these phenomena are very interesting. This work gives an explanation of the enhancement effect based on elastic dipole theory and shows that by analysing the dynamical processes one can obtain two diffusion activation energies of charged defects within the crystal from the intensity relaxation curve, a new method which has not been used before.

1. Explanation. $-\alpha-\mathrm{LiIO}_{3}$ crystal is a nearly onedimensional ionic semiconductor with space group $C_{6}^{6}[4,5]$. The positive and negative charged defects, which are distributed almost homogeneously throughout the crystal with a concentration of about $10^{-5}$ (estimated from the amount of impurities in the crystal determined by mass-spectroscopic analysis) before the application of the external field, will start to migrate towards both electrodes under the influence of the external field; during this process some of the defects will be stopped and will thus precipitate around macroscopic imperfections (layer-like defects, dislocations...), but most of them will, finally, accumulate in a small region directly under the electrodes. The defect concentration gradient near an electrode at a depth of about $10^{-2} \mathrm{~cm}$ could approach a value of around $3 \times 10^{-3} / \mathrm{cm}$. (This can be estimated from the charge density of 1 electronic charge $/ 10^{6}$ unit cell, a value obtained by the "freezing effect» experiment [1].) According to elastic dipole theory [6] the relative change of the lattice constant is proportional to the defect concentration $C$ when $C \leqslant 10^{-3}$. Thus, if different kinds of defects accumulate under the electrode, the ratio of the lattice constant gradient to the lattice constant $(\operatorname{grad} d) / d$ in this region can easily be 
estimated as $\sim 1 \times 10^{-3} / \mathrm{cm}$ (induced by the vacancies of $\mathrm{Li}^{+}$cations) and $\sim 1 \times 10^{-2} / \mathrm{cm}$ (induced by interstitial $\mathrm{Li}^{+}$cations). The existence of a lattice gradient will broaden the angular and energy range which satisfy the Bragg-diffraction condition and thus will enhance the neutron diffraction intensity. In our case the parameters are as follows : $d_{002}=2.58 \AA$, $\lambda_{\mathrm{n}}=1.08 \AA, \theta_{\mathrm{B}}=12^{\circ} 5^{\prime}$, structure factor

$$
F_{002}=3.06 \times 10^{-12} \mathrm{~cm},
$$

which give the extinction thickness for neutrons in the (002) case, $t_{\text {ext }}=1.72 \times 10^{-3} \mathrm{~cm}$. From the simple model of neutron dynamical diffraction for a deformed crystal [7], the total enhancement value can be calculated by considering the crystal as being composed of many thin layers and by evaluating the total result from the reflection within each layer; this gives an enhancement factor $\left(I-I_{0}\right) / I_{0}=310 \%$ at a field strength of about $100 \mathrm{~V} / \mathrm{cm}$; this value agrees rather well with the experimental results.

2. Evaluation of the diffusion activation energy of defects within $\alpha$ - $\mathrm{LiIO}_{3}$ from the measured enhancement relaxation curve. - In the previous section the steady state diffraction enhancement due to the migration of charged defects within the crystal in a DC field has been analysed. We now proceed to consider the relaxation process itself. Since both positive and negative charged defects participate in the conduction process, the migration behaviour of the defects can be described by the Debye-Hückel equation and the Poisson equation when a DC field is applied to the crystal. Fatuzzo and Coppo [8] showed that, when both electrodes are fully blocked by defects, the solution of these equations near the electrodes can be written approximately as

$$
\begin{aligned}
C(z, t)=C_{0}(z)+\Delta C(z, 0) \exp (-t / \tau)+ \\
+\Delta C_{\infty}[1-\exp (-t / \tau)]
\end{aligned}
$$

where $C(z, t)$ is the concentration of positively or negatively charged defects at position $z$ and time $t$, $C_{0}(z)$ is the concentration distribution within the crystal before the application of the external field, which can be assumed to be constant in the following equation, and $\Delta C(z, 0)$ and $\Delta C_{\infty}(z)$ are the increments of the concentration distribution within the crystal after the application of the external field at $t=0$ and $t=\infty$ respectively. Since $\Delta C(z, 0) \ll \Delta C_{\infty}(z)$, the second term of equation (1) can be omitted in comparison with the third term. $\tau$ is the relaxation time constant which derives from the migration of defects between the two electrodes.

In the case of a $\alpha-\mathrm{LiIO}_{3}$ monocrystal, if there are both positive and negative gradients induced from positive defects and negative defects respectively near the anode at a depth of about $10^{-2} \mathrm{~cm}$ after application of the DC field, then equation (1) can be converted into the form of equations (2), (3), in which the origin of the coordinates is chosen at the surface of the anode $\left({ }^{1}\right)$ :

$C_{\mathrm{p}}(z, t)=C_{\mathrm{p} 0}+D_{\mathrm{p} \infty}\left(1-10^{2} z\right)\left[1-\exp \left(-t / \tau_{\mathrm{p}}\right)\right]$.

$C_{\mathrm{n}}(z, t)=C_{\mathrm{n} 0}+D_{\mathrm{n} \infty}\left(1-10^{2} z\right)\left[1-\exp \left(-t / \tau_{\mathrm{n}}\right)\right]$

where $D_{\mathrm{p}_{\infty}}<0, D_{\mathrm{n}_{\infty}}>0$ and $z \leqslant 10^{-2}$.

Here $C_{\mathrm{p}}$ and $C_{\mathrm{n}}$ represent, respectively, the concentration of positive and negative charged defects. The coexistence of equations (2) and (3) means that the layer immediately under the anode is both the accumulation layer for negative charged defects and the depletion layer for positively charged defects.

Let us consider the neutron diffraction case for which the (002) plane of $\alpha-\mathrm{LiIO}_{3}$ is parallel to the surface of the electrode. From the neutron dynamical diffraction theory for deformed crystals $[7,9]$ it can easily be shown that the enhancement factor $\left(I-I_{0}\right) / I_{0}$ is proportional to the lattice constant gradient provided that the deformation is small

$$
\begin{gathered}
\left(\left(\operatorname{grad} d_{002}\right) / d_{002}<1 \times 10^{-2} / \mathrm{cm}\right) \text { i.e. }, \\
\frac{I-I_{0}}{I_{0}}=A \frac{\partial d_{002}}{\partial z} .
\end{gathered}
$$

As we have mentioned in the previous section, the lattice constant gradient in the crystal immediately under the electrode is induced by the gradiert of the positive or negative defect concentration. Since the elastic dipole theory shows that the deformation of host lattice due to different kinds of defects is different (for example, the lattice constant can be reduced by vacancies of $\mathrm{Li}^{+}$cations and can be increased by the interstitial $\mathrm{Li}^{+}$cations) we have

$$
\frac{\partial d_{002}(z, t)}{\partial z}=K_{\mathrm{p}} \frac{\partial C_{\mathrm{p}}(z, t)}{\partial z}+K_{\mathrm{n}} \frac{\partial C_{\mathrm{n}}(z, t)}{\partial z}
$$

where $K_{\mathrm{p}}>0, K_{\mathrm{n}}<0$.

Thus the enhancement factor of the neutron diffraction intensity can be written as

$$
\begin{aligned}
\frac{I-I_{0}}{I_{0}}=A\left[K_{\mathrm{p}} \frac{\partial C_{\mathrm{p}}(z, t)}{\partial z}+K_{\mathrm{n}} \frac{\partial C_{\mathrm{n}}(z, t)}{\partial z}\right] \\
=-10^{2} A K_{\mathrm{p}} D_{\mathrm{p} \infty}\left[1-\exp \left(-t / \tau_{\mathrm{p}}\right)\right]- \\
\quad-10^{2} A K_{\mathrm{n}} D_{\mathrm{n} \infty}\left[1-\exp \left(-t / \tau_{\mathrm{n}}\right)\right]
\end{aligned}
$$

( ${ }^{1}$ ) In fact, equation (4) can also be evaluated if the 2 nd term in equations (2) and (3) takes the form, $D_{\mathrm{p} \infty}(z)[1-\exp (-t / \tau)]$, and if $D_{\mathrm{p} \infty}=B \times \exp (-\alpha z)$ where $\alpha \sim 50 \mathrm{~cm}^{-1}$ (the most probable case). Thus the constant gradient assumption is not necessary and is only taken for convenience. 
or the intensity relaxation will be

$$
\begin{aligned}
I=I_{0}+I_{1}\left[1-\exp \left(-t / \tau_{1}\right)\right]+ & \\
& +I_{2}\left[1-\exp \left(-t / \tau_{2}\right)\right]
\end{aligned}
$$

where $\tau_{1}$ and $\tau_{2}$ are the relaxation time constants due to two different kinds of defects.

Physically this means that the enhancement factor is proportional to the gradient of the defect concentration under the electrodes. This is an important result, because it directly relates the experimentally measured enhancement relaxation to the relaxation behaviour of the defect concentration gradient under the electrode.

Furthermore, keep in mind that both $\tau_{1}^{-1}$ and $\tau_{2}^{-1}$ in equation (4) are proportional to the product of the rate of migration of defects, $\mu$, and the defect concentration, $C_{0}$, so that $\tau$ can be written as

$$
\begin{aligned}
& \tau_{1}^{-1}=\tau_{10}^{-1} \exp \left(-U_{1} / K T\right) \\
& \tau_{2}^{-1}=\tau_{20}^{-1} \exp \left(-U_{2} / K T\right)
\end{aligned}
$$

where $U_{1}$ and $U_{2}$ are the diffusion activation energies of the defects. We can obtain the relaxation time constants $\tau_{1}\left(T_{j}\right), \tau_{2}\left(T_{j}\right)(j=1,2, \ldots, M)$ for these two kinds of defects at $M$ different temperatures by fitting the experimental $\left(I / I_{0}, t\right)$ curve with equation (4). Then, by the linear relation between $\log 1 / \tau_{1}$ or $\log 1 / \tau_{2}$ and $1 / T$ one can determine the diffusion activation energy for the two different kinds of defects within the crystal simultaneously and independently

As an example, one of the two sets of enhancement relaxation curves obtained by Yang Zhen et al. [1] for different temperatures can be analysed as follows : figure 1 and figure 2 are theoretical fits of the experimental results (relaxation curve in figure 5 of [1]; field strength $24 \mathrm{~V} / \mathrm{cm}$, temperature $283 \mathrm{~K}$, $326 \mathrm{~K}$ and $305 \mathrm{~K}, 344 \mathrm{~K}$ ). The solid line is a fit using equation (4) with two exponents; the $\tau_{1}, \tau_{2}$ value are listed in table $I$, in which the $I_{1 \infty} / I_{0}, I_{2 \infty} / I_{0}$ and $I_{2 \infty} / I_{1 \infty}$ value are also included. It can be seen that

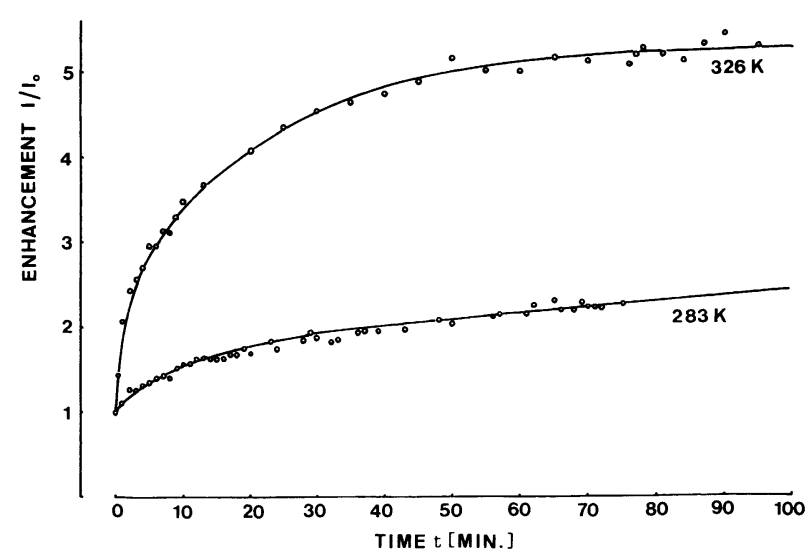

Fig. 1. - Theoretical fit of the relaxation curve. $O$ experiment points ; theoretical fit using equation (4) and $\tau_{1}, \tau_{2}, I_{1 \infty} / I_{0}$, $I_{2 \infty} / I_{0}$ listed in table $\mathrm{I}$.

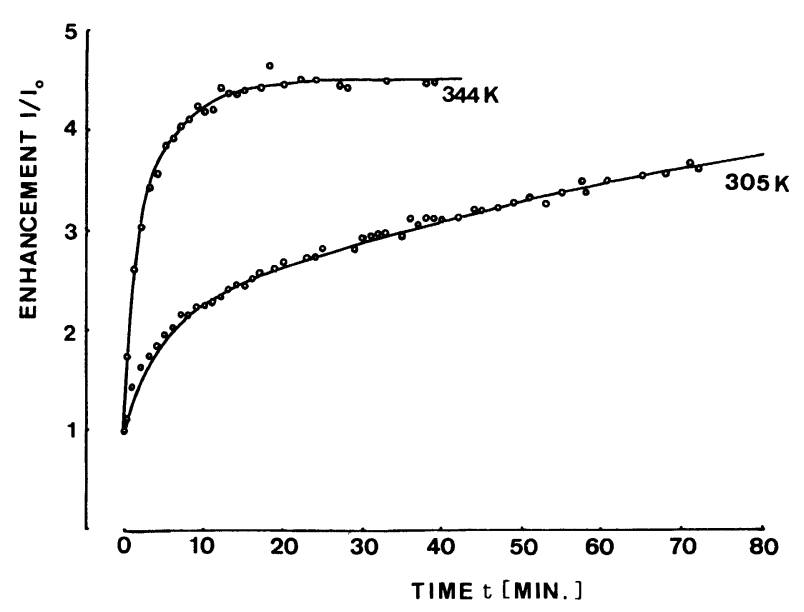

Fig. 2. - Same as figure 1 but at two further different temperatures.

Table I. - Parameters used for the theoretical fitting of figures 1 and 2.

$\begin{array}{cccccc}T & \tau_{1} & \begin{array}{c}\tau_{2} \\ \text { (min.) }\end{array} & I_{1 \infty} / I_{0} & I_{2 \infty} / I_{0} & I_{2 \infty} / I_{1 \infty} \\ (\mathrm{K}) & \text { (min.) } & - & - & - & - \\ \overline{283} & 10.0 & 386 & 0.72 & 3.17 & 4.40 \\ 305 & 4.02 & 86.6 & 1.04 & 2.84 & 2.73 \\ 326 & 1.48 & 22.5 & 1.30 & 3.04 & 2.34 \\ 344 & 0.924 & 5.70 & 1.82 & 1.70 & 0.93\end{array}$

the fit is rather good, further confirming that the change in value of the lattice constants near the electrodes is promoted by two kinds of defects. Note that the value of $I_{2 \infty} / I_{1 \infty}$ listed in table $I$ is different at different temperatures : this probably reflects the change in the concentration of the two kinds of defects at these temperatures.

The second step of the analysing process is to draw the $\log \tau^{-1}--T^{-1}$ curve as already mentioned. This is shown in figure 3 ; from the slope of the two welldefined straight lines, two different activation energies of $\alpha-\mathrm{LiIO}_{3} U_{1}=0.35 \pm 0.4 \mathrm{eV}$ and

$$
U_{2}=0.58 \pm 0.5 \mathrm{eV}
$$

can easily be obtained $\left({ }^{2}\right)$. These results can be compared with values obtained by other methods, e.g., $U_{1}$ of $0.36 \mathrm{eV}$ by $50 \mathrm{~Hz}$ AC conductivity (Haüssuhl [10]), $\mathrm{U}_{2}$ of $0.50-0.58 \mathrm{eV}$ and $0.57-0.66 \mathrm{eV}$ by DC conductivity (Remoissenet et al. [11], Zhu Yong et al. [12]); the agreement seems satisfactory.

From the activation energy and $I_{2 \infty} / I_{1 \infty}$ at different $T$ obtained by this new method, one can tentatively assume that the conductive defects for $\alpha-\mathrm{LiIO}_{3}$ are Frenkel pairs of $\mathrm{Li}^{+}$cation in which

(2) In fact the second set of data has also been analysed by this method and an activation energy of $U=0.32 \mathrm{eV}$ was obtained; the time scale of the experimental data is too short for determination of the second activation energy. 


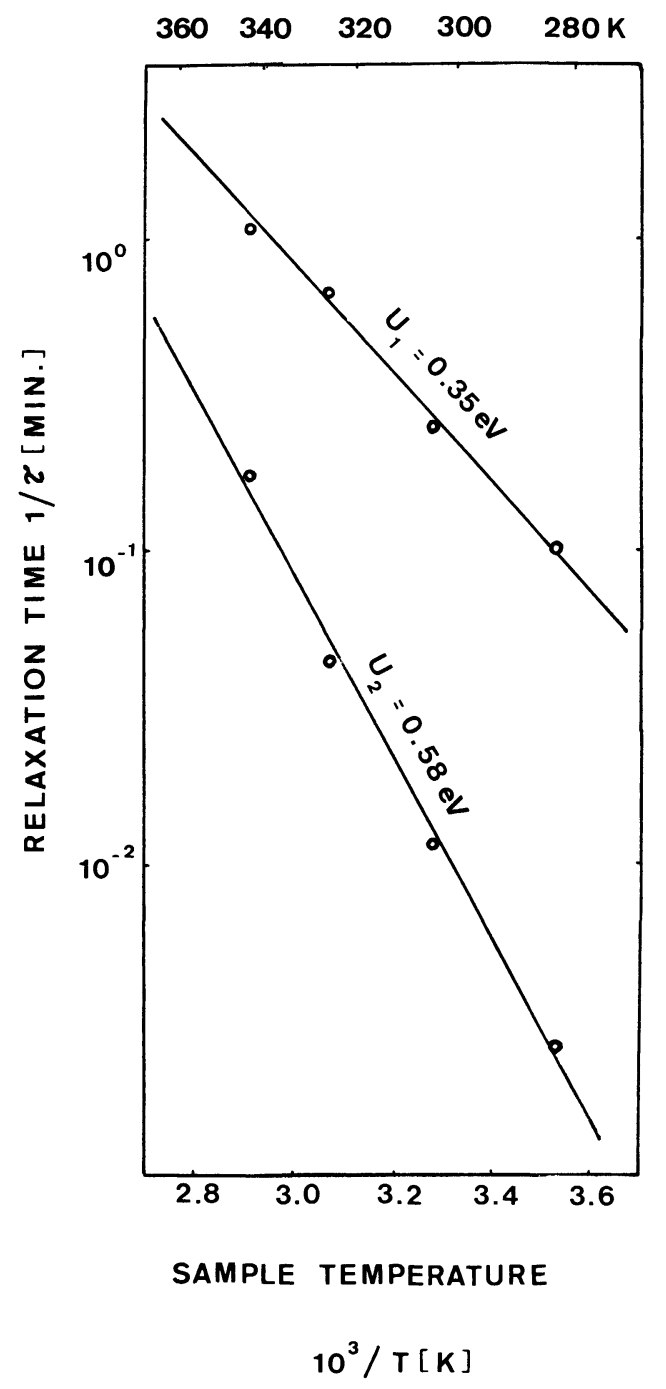

Fig. 3. - Log $1 / \tau v s .1 / T$ curve as predicted in equations (5) and (6), all points group into two straight lines.
$U_{2}=0.58 \mathrm{eV}$ represents the jump activation energy, $H_{\mathrm{m}}^{\mathrm{v}}$, for the vacancy of one $\mathrm{Li}^{+}$cation, and $U_{1}=0.36 \mathrm{eV}$ is equivalent $(G / 2)+H_{\mathrm{m}}^{\mathrm{i}} ; G$ is the free energy of formation of one Frenkel pair. $H_{\mathrm{m}}^{\mathrm{i}}$ is the jump activation energy of one $\mathrm{Li}^{+}$cation.

3. Discussion. - The strong anisotropy observed in the experiment (no enhancement in the $(h k 0)$ group of planes) is probably due to the large $\left(\operatorname{grad}_{z} d_{00 l}\right) / d_{00 l}$ and small $(\sim 0)\left(\operatorname{grad}_{z} d_{h k 0}\right) / d_{h k 0}$ value resulting from clustering of the point defects within the hexagonal basal plane of this crystal [13].

The frequency dependence of this effect can be explained as being due to a finite value of the migration rate of the charged defects, which cannot follow the fast-changing external field at high frequency.

\section{References}

[1] Yang Zhen, Niu Shiwen and Cheng Yufen, Scientia Sinica XX 11 (1979) 1000.

[2] Yang Cheng, Cheng Yufen and Niu Shiwen, Acta Phys. Sinica 24 (1975) 6.

[3] CAHN, R. W., Nature 282 (1979) 359.

[4] De Boer, J. L., Van Bolhuis, F., Roeli Olthof-Hazekamp and AAFJE Vos, Acta Crystallogr. 21 (1966) 841.

[5] Arlt, G., Puschert, W. and Quadflieg, P., Phys. Status Solidi 3 (1970) K 243.

[6] PeisL, H. in Defects and Their Structure in Nonmetallic Solids, ed. by Henderson B. and Hughes A. E. (Plenum Press, New York) 1975, p. 381.

[7] Albertini, G. and Bceuf, A., Acta Crystallogr. A 32 (1976) 863.

[8] Fatuzzo, E. and Coppo, S., J. Appl. Phys. 43 (1972) 1457.

[9] TAupin, D., Bull. Soc. Fr. Mineral. Crystallogr. 87 (1964) 469.

[10] Haüssuml, S., Phys. Status Solidi 29 (1968) K 159.

[11] Remoissenet, M., Garandet, J. and Arend, H., Mat. Res. Bull. 10 (1975) 181.

[12] Zhu Yong, Zhang Dao-fan and Cheng XI-min, Acta Phys. Sinica 26 (1977) 115.

[13] Ehrhart, P. and Schönfeld, B., Phys. Rev. B. 19 (1979) 3896. 